\title{
Use of Satellite Imagery at the ICRC (International Committee of the Red Cross)
}

\author{
Yann Rebois and Friderieke Alschner \\ International Committee of the Red Cross, Geneva/Switzerland·gisupport@icrc.org
}

Short paper

\begin{abstract}
The International Committee of the Red Cross (ICRC) has been using satellite imagery for more than 10 years now; initially for hydrogeological analysis in arid and semi-arid areas, as well as for vegetation follow-up in agricultural projects (irrigation, seeds distributions, etc.).

Today, the humanitarian organization most frequently uses very high-resolution imagery for urban water projects, where the necessity to identify population densities, water resources, water supply needs, and pipeline routes is relevant. A growing field of interest is the use of imagery to analyze hostilities during conflict situations.

Data and imagery is hosted on our servers enabling our field staff to access them from any of our field offices through web clients from ICRC's intranet. This approach allows the sharing of simple PDF-maps, but also web-maps, web-applications, or entire dashboards within the ICRC, depending on the needs.

\section{A Constant Use of Earth Observation for the Operations with a Shift to the "Protection of Civilian Population" due to the Increasing Spatial Resolution of the Images}

The International Committee of the Red Cross (ICRC) is one of the largest humanitarian organizations. The organization provides assistance and protection to the victims of war. In terms of assistance, the institution delivers water and habitat support, food security, and health services. The primary use of satellite images has always been done for the management of an operation. Until today, it is mainly used by the Water and Habitat Unit. The ICRC mostly uses pre-processed imagery from main commercial satellite image providers like DigitalGlobe or Astrium. Several projects were conducted by assessing population numbers according to dwellings, and land use, based on a visual analysis of the imagery, in order to assess and improve water networks in urban centers like Mazar-e-Sharif, Bassikounou, or Ndelé. For such projects, available data is mostly out of date or simply nonexistant. Using satellite imagery, digitizing and extracting the relevant information allows the ICRC to build a timely response based on recent information. The ICRC sometimes reaches out to the community of the digital volunteers, like OpenStreetMap (OSM), for tasks such as digitizing images. 
Within ICRC, GIS is now becoming a transversal tool, benefitting various units, and its user group is constantly growing. Lately, ICRC's Protection Division realized the potential of remote sensing data and analysis for the purposes of protecting civilian population and promoting and monitoring human rights such as already done by UNOSAT or Human Rights Watch. The Logistic Division now has a focal point working with logistic data including its spatial implications. This increase in needs and efforts in the field of GIS by various units has to be answered by an organizational strategy. Mechanisms and tools must be put in place in order to avoid duplicated efforts in data collection and visualization or inconsistent data between units.

The analysis of the satellite images is slowly starting. For the latest events like in Gaza, South Sudan, Syria, and Ukraine, or typhoon Hagupit, image interpretation helped measure damages and impact on the civilian population and therefore allowed the ICRC to adapt its humanitarian response accordingly. Such analysis is mainly done on buildings and infrastructure facilities, from a "simple, burnt tukul" where the damage identification is fairly easy (figure 1) to more challenging areas like in urban neighborhoods.

The image analysis is associated with knowledge from the field and local GIS datasets in order to grasp the first needs for the civilian population, and then for reporting on the potential violation of the humanitarian law to the actors of the conflict.
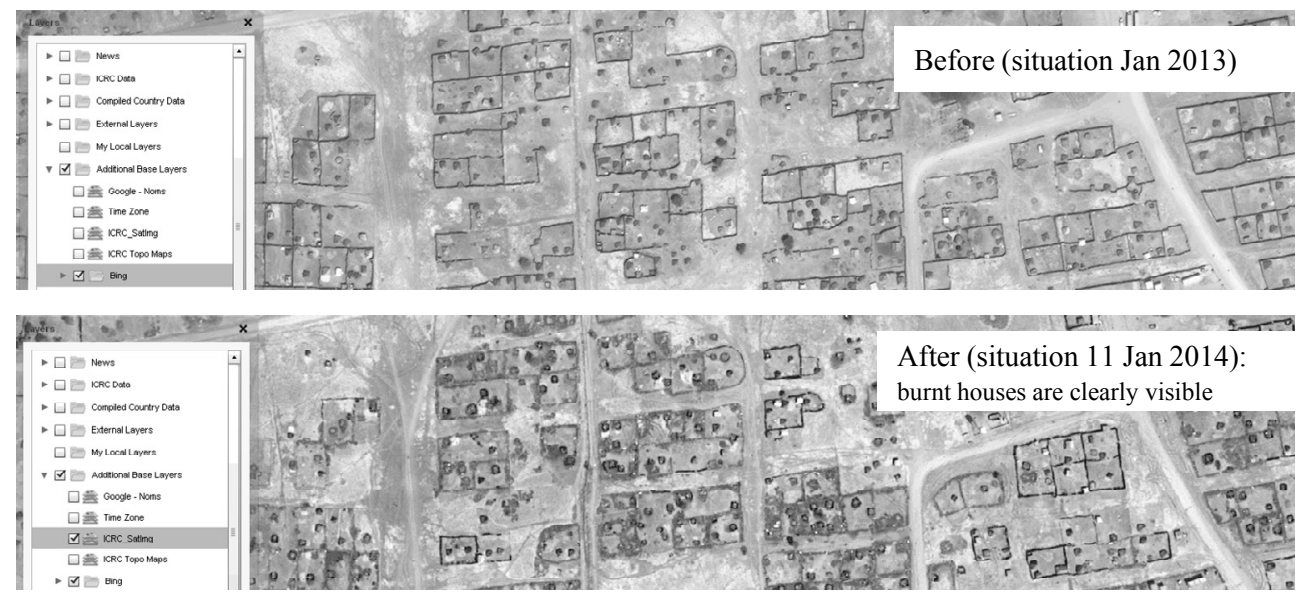

Fig. 1: Sample of damage analysis done in early 2014 in a settlement in South Sudan. Estimation of burnt houses according to their structure (grass roof/versus concrete). Analysis was done based on a Pléiades image.

\section{Sharing Data and Analysis within the ICRC, Allowing Access as Key for Feedback and Field Validation of Analysis}

Due to the requirement of a speedy response, quantified analysis is done using visual image interpretation. We might reach out to OSM for digitization or do internal crowd sourcing where data might be sensible. This is possible due to a strong GIS infrastructure based on 
an ArcGIS server and an internal geoportal. Once the analysis is done, depending on data sensibility, the images, maps, associated data and other GIS-products related to the crisis will be shared with the different users from the field to the Headquarters. It is one of the key challenges to ensure accessibility to the raw image, to the analysis, and to the contextual information aggregated by the local Information Manager/GIS Officer to the top manager. After processing the image and finalizing the analysis, the image can be made available through the internal geoportal. Furthermore, the GIS team will provide easy to share paper maps, or, if requested, web-applications (figure 2) displaying available paper-maps, as well as an interactive web-map allowing the user to have an overview of all available products and results and to interact with the data. Those products, accessible only for internal staff members, allow the staff to get insights into a situation before being deployed, but also to give feedback on the analysis after visiting a place, which is key for the validation of each analysis.
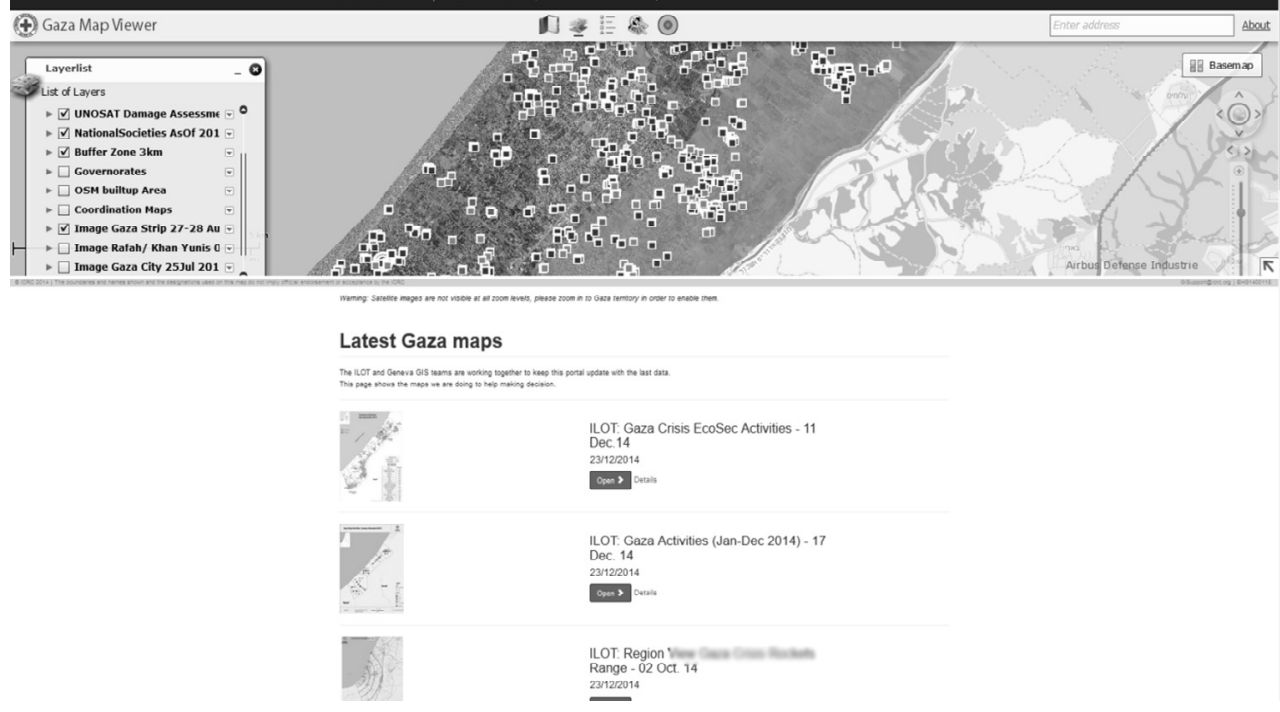

Fig. 2: Example of Operational Portal mixing web content from humanitarian actors, available geodata, ICRC data and satellite imagery over the Gaza strip. Static maps related to the crisis are also available off the shelf.

Another example could be provided during the crisis in South Sudan with the displaced population camp in Bentiu, January 2014 (figure 3): 14000 Internally Displaced Persons (IDPs) were supposed to be in the United Nations Mission in South Sudan (UNMISS) compound. The ICRC provided primary help to the displaced population such as drinkable water and food and non-food items. To do so, an estimate of the population numbers was required, in order to ensure meeting the needs of the population. 


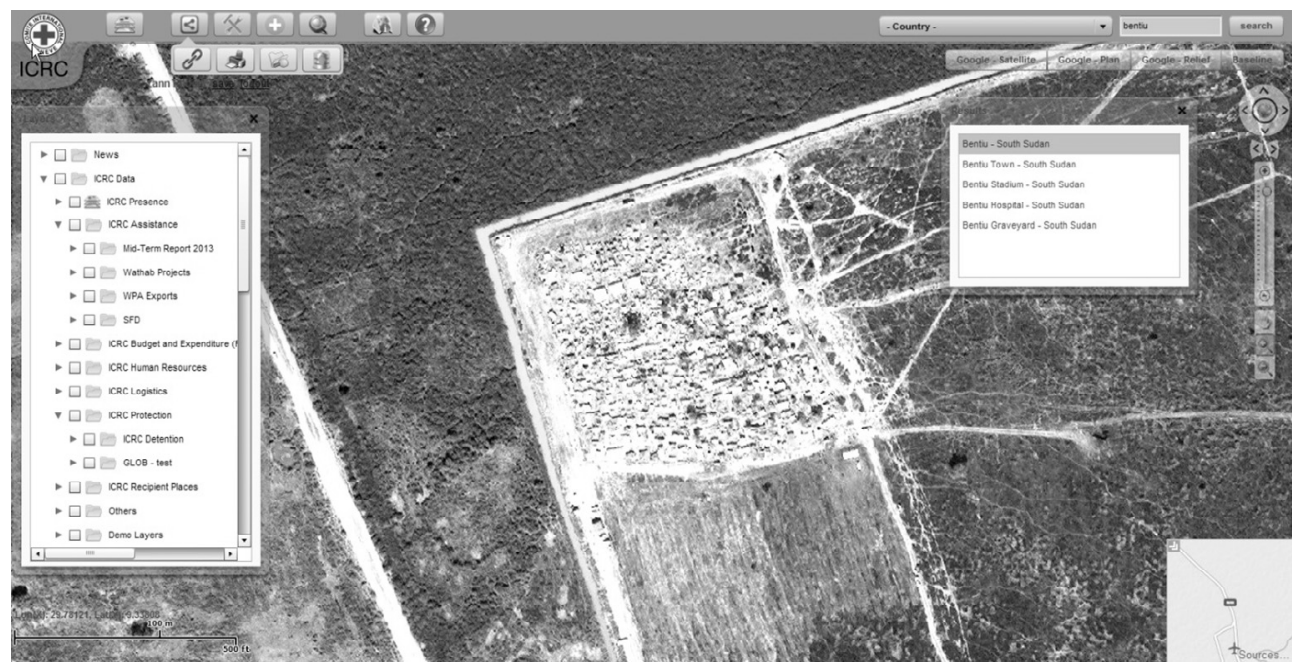

Fig. 3: Imagery of UNMISS compound in Bentiu. Image displayed in the ICRC Geoportal. Comparison of supposed IDP figures with population estimated after assessment based on satellite imagery.

Based on the visual analysis of the satellite imagery we knew that the camp area is around $25,000 \mathrm{~m}^{2}$, with at least $50 \%$ of "not built-up" space and a high concentration of tents in the camp was not visible. Therefore assuming $4 \mathrm{~m}^{2}$ of shelter per person, we estimated that there are between 1500 and 3000 IDPs in Bentiu.

Sharing the result of our analysis, which diverged strongly from the number of 14000 IDPs spread earlier within the humanitarian community, with the field worker, he replied:

"I had the same doubts myself these last days. Actually, our truck had problems delivering $60 \mathrm{~m}^{3}$ of water yesterday (3 rotations). There were still 2000 liters in the truck at 6pm and no one queuing to get water... Anyway, the IDPs are extremely satisfied with the amount and quality of the water delivered". As said by Marc.

\section{Conclusions and Outlook}

A good analysis of the situation is key to adjust the ICRC's response, and make sure that aid is delivered where it is most needed. It helps the different units to prioritize the ICRC humanitarian response. Working with the satellite images helps in identifying patterns of violation and their perpetrators. Furthermore, it helps the team on the ground in determining the extent of civilian casualties/civilian loss per violation. Satellite imagery can help achieve this. Therefore, the analysis of satellite imagery will play an important role and its use might continue to increase in the future within the ICRC. 